\title{
La funduplicatura laparoscópica mejora la calidad de vida en pacientes con reflujo gastroesofágico
}

\author{
Laparoscopic funduplicature improves quality of life in patients with gastro-esophageal reflux
}

Grant A, y col. BMJ. 2008; 337:a2664

\section{Objetivo}

Comparar la funduplicatura laparoscópica con el tratamiento médico en pacientes con enfermedad crónica por reflujo gastroesofágico (ERGE).

\section{Diseño, lugar y pacientes}

Ensayo clínico multicéntrico pragmático* (incluyó un grupo en que la intervención evaluada fue aleatorizada y otro, en la que no lo fue) llevado a cabo en 21 hospitales del Reino Unido, que incluyó 1078 pacientes con más de un año de enfermedad documentada por endoscopía o "pHmetría", bajo riesgo quirúrgico y ausencia de esófago de Barret o displasia mayor a $3 \mathrm{~cm}$, hernia paraesofágica, estenosis esofágica u obesidad mórbida. El seguimiento fue de 12 meses e incluyó 357 pacientes aleatorizados a tratamiento médico optimizado o funduplicatura, y un grupo paralelo $(n=453)$ no aleatorizado que eligió su tratamiento.

\section{Medición de resultados principales}

El resultado primario fue calidad de vida (puntaje de 0 a 100 en el cuestionario Reflux QoL1) medido al ingreso, a los tres y a los 12 meses luego de la intervención. A mayor puntaje, mejor calidad de vida, considerándose clínicamente relevante una diferencia de siete puntos. El análisis fue por intención de tratar.

\section{Resultados principales}

Se resumen en la tabla 1 . La tasa de complicaciones quirúrgicas fue de $0,6 \%$ y no se registraron muertes.
Tabla 1: calidad de vida relacionada con los síntomas de reflujo -basal y a los 12 meses de la intervención- de acuerdo a la rama de tratamiento asignada aleatoriamente y con análisis por intención de tratar.

\begin{tabular}{|c|c|c|c|}
\hline \multirow{2}{*}{$\begin{array}{l}\text { Puntaje de severidad } \\
\text { del reflujo }\end{array}$} & \multicolumn{2}{|c|}{ Rama de tratamiento } & \multirow{3}{*}{$\begin{array}{c}\text { Diferencia de } \\
\text { puntaje a los } 12 \\
\text { meses } \\
\text { (IC95\%) }\end{array}$} \\
\hline & $\begin{array}{c}\text { Quirúrgico } \\
(n=178)\end{array}$ & $\begin{array}{l}\text { No quirúrgico } \\
(n=179)\end{array}$ & \\
\hline Basal & 63,6 & 66,8 & \\
\hline A los 12 meses & 84,6 & 73,4 & 11,2 (6,4 a 16) \\
\hline
\end{tabular}

${ }^{a}$ A mayor puntaje, mejor calidad de vida.

Respecto del grupo no aleatorizado $(n=453)$ los pacientes que eligieron cirugía tuvieron puntajes basales más bajos y luego de hacer un ajuste por índice de masa corporal, edad, sexo y puntaje basal, los resultados fueron estadísticamente favorables al grupo que eligió cirugía con una diferencia de 4,1 puntos.

A los 12 meses y considerando globalmente a ambos grupos, recibía inhibidores de la bomba de protones (IBP) el $38 \%$ de los pacientes operados y el $90 \%$ de los asignados a tratamiento médico. La tasa de complicaciones quirúrgicas fue de 0,6\% y no se registraron muertes.

\section{Conclusiones}

A los 12 meses, la cirugía mejoró el puntaje de calidad de vida en los pacientes con ERGE.

Palabras claves: Enfermedad por reflujo, funduplicatura, medicación antireflujo, calidad de vida. Keywords: gastro-oesophageal reflux disease, fundoplication, antireflux medication, quality of life. Fuentes de financiamiento: no referida.

\section{Comentario}

A los pacientes con ERGE sin complicaciones graves (estenosis o esófago de Barret) sólo se les ofrecía la opción quirúrgica ante la progresión de sus síntomas a pesar del tratamiento médico ${ }^{12}$. Utilizando un diseño pragmático cercano a la práctica habitual, este estudio evaluó pacientes a quienes habitualmente no se les indicaría funduplicatura como tratamiento inicial.

Sus resultados favorecieron la estrategia quirúrgica inicial, aunque llama la atención que el $38 \%$ de los pacientes operados hayan continuado con requerimientos de medicación antireflujo al año de seguimiento. En este sentido, vale destacar que varios ensayos clínicos habían cuestionado los beneficios a más largo plazo de la cirugía para el control de la ERGE. Por ejemplo, el de Lundell y col. ${ }^{2}$ había documentado que a los cinco años, los pacientes operados utilizaban la misma cantidad de medicación anti-reflujo que los no operados; mientras que el de Spechler y col. $^{3}$ había reportado que a los diez años, el $62 \%$ de los pacientes operados seguían consumiendo medicamentos antirreflujo.

Esto indicaría que con el paso del tiempo, los beneficios atribuibles a la cirugía se van perdiendo.

\section{Conclusiones de la comentadora}

La funduplicatura laparoscópica constituye un procedimiento breve, con escaso riesgo, y que se asocia a una mejoría de la calidad de vida al año de los pacientes con ERGE no complicado. Sin embargo, ya que la evidencia sobre sus beneficios a largo plazo es controvertida, la indicación de cirugía debería ser evaluada en cada paciente en particular.

Tami Guenzelovich y Victoria Wurcel [ Servicio de Medicina Familiar y Comunitaria. Hospital Italiano de Buenos Aires. tami.guenzelovich@ hospitalitaliano.org.ar ]

Guenzelovich T y Wurcel V. La funduplicatura laparoscópica mejora la calidad de vida en pacientes con reflujo gastroesofágico. Evid. actual. práct. ambul; 12 (4): 128, Oct-Dic.2009. Comentado de Grant AM et al. Minimal access surgery compared with medical management for chronic gastro-esophageal reflux disease: UK collaborative randomised trial. BMJ 2008;337:a2664.PMID: 19074946.

\section{Referencias}

1. Macran S y col. The development of a new measure of quality of life in the management of gastro-esophageal reflux disease: the Reflux questionnaire. Qual Life Res 2007; 16:331-43, 2. Lundell $\mathrm{L}$ y col. Continued (5-year) followup of a randomized clinical study comparing antireflux surgery and omeprazole in gastroesophageal reflux disease. J Am Coll Surg. Feb 2001;192(2):172-9; discussion 179-81

3. Spechler J y col. Long- term outcome of medical and surgical therapies for gastroesophageal reflux disease: follow up of a randomized controlled trial. JAMA 2001; 285:2331-8. 